rheumatoid arthritis with and without extraarticular features and SLE in Northern Italy. $J$ Rheumatol 1988; 15: 51-3.

5 Ben-Cherit E, Brautbar C, Rubinov A. HLA antigens in Reiter's syndrome in Israeli patients. $J$ Rheumatol 1985; 12: 964-6.

6 Savi M, Ferraccioli G F, Neri T M, et al. HLA-DR antigens and anti-cardiolipin antibodies in Northern Italian SLE patients. Arthritis Rheum (in press).

\section{Anticardiolipin versus lupus anticoagulant tests: no final judgment}

SIR, I think it important to correct some statements made in a recent paper by Derksen and colleagues. ${ }^{1}$ Their study was a correlation of various lupus anticoagulant tests and their anticardiolipin (aCL) test with thrombosis and fetal loss in 111 lupus patients. In their discussion they implied that their group with systemic lupus erythematosus (SLE) was similar to the population of patients reported by our group in a previous study. ${ }^{2}$ This is incorrect. Our study looked at 121 patients with varying levels of $\mathrm{aCL}$ antibodies irrespective of underlying diagnosis. One third of the patients had normal aCL levels and only about $60 \%$ of the high positive group had SLE. The high percentage of patients with thrombosis and fetal loss in our report was largely influenced by the number of high $\mathrm{aCL}$ positive patients with these complications. Now that standard sera are available, and with improvements in the aCL test, ${ }^{3}$ our own and other reputable groups with similar experience continue to find that moderate to high positive aCL test results will identify a population (both SLE and non-SLE) with a high incidence of thrombosis and fetal loss. This statement will be tested further in a prospective study planned by the Kingston antiphospholipid study (KAPS) group. ${ }^{4}$

Dr Derksen also stated that 'sera exchanged between his laboratory and that of the Lupus Research Laboratory were concordantly classified as negative, low, medium, or high positive for anticardiolipin antibodies'. Dr Derksen participated in the International Anticardiolipin Standardisation Workshop and had a valid assay, but this need not imply in any way concordance of test results between his laboratory and our own. Since the standardisation workshop there has been no formal study conducted between Dr Derksen's laboratory and our own that involved an exchange of sera and tests for concordance of our results. Indeed, such a process is only now being attempted at an international level by the KAPS group. In reviewing Dr Derksen's aCL assay method I must emphasise that the particular units and serum dilutions he used were his own and not those of the international workshop. ${ }^{3}$ In addition, in assays performed by the Lupus Research Laboratory (and now by my own laboratory in Louisville) the cut off level of low positive results (20 GPL or $20 \mathrm{MPL}$ units) is well above 2SD of normal controls. Despite our efforts to standardise the aCL test my experience suggests that concordance between laboratory results in the absolute terms used by $\mathrm{Dr}$ Derksen will prove a difficult goal indeed.
In 1983 Doctors Gharavi and Hughes and I made the decision to set up an anticardiolipin test after it was found that lupus anticoagulant tests performed routinely in our $\bar{O}$ hospital were not sufficiently sensitive and reproducible to $\frac{\bar{\sigma}}{\mathrm{C}}$ identify patients whom we believed to have the lupus $\overline{\bar{\omega}}$ anticoagulant syndrome. ${ }^{5}$ Over the last five years and in $\overrightarrow{\mathbb{D}}$ the course of working in three very different institutions I $\varrho$ remain convinced that our original decision was correct. We have managed to identify a substantial number of $\vec{\circ}$ patients using the aCL test alone. My most recent experience in Louisville, Kentucky is a case in point. My laboratory has measured aCL levels in about 300 sera and found nine sera with moderate to high positive results. Seven of these sera were from patients with recurrent fetal loss or thrombosis, or both. Despite the fact that these $\stackrel{\oplus}{\infty}$ patients had markedly raised IgG or IgM concentrations $\overrightarrow{-}$ on several occasions I obtained a positive lupus anti- $\infty$ coagulant test result on only two occasions. It is probable 0 that the lupus anticoagulant test performed by the $\vec{J}$ laboratory to which we send our plasma has an insensitive $\overrightarrow{\vec{c}}$ test. In busy outpatient settings in large institutions it is practically impossible for clinicians to meet the technicians performing coagulation tests, much less to have technicians perform the numbers of tests for the lupus anticoagulant outlined by Derksen and colleagues. The requirements $\oplus$ that plasma specimens be platelet-poor and be freshly $\mathscr{\oplus}$ prepared (or stored at $-70^{\circ} \mathrm{C}$ ) impose further constraints. In addition, standardisation of the lupus anticoagulant test and measurement of these test results are difficult tasks and are only now being addressed by some international groups, including the KAPS group.

At a time when we are still improving both solid phase antiphospholipid tests and standardising lupus anti- $\overrightarrow{\vec{\sigma}}$ coagulant tests sweeping statements such as that made by 3 Derksen and colleagues in the title of their paper are $\vec{\partial}$ premature. The recent creation of the KAPS group in Jamaica was designed to address some of the issues raised by Derksen and colleagues. Until we know better, wisdom suggests that both tests be performed, if possible.

Division of Rheumatology,

E NIGEL HARRIS

Department of Medicine,

University of Louisville,

Louisville,

Kentucky 40292,

USA

\section{References}

1 Derksen R H W M, Hasselaar P, Blokzijl L, Gmelig Meyling F H J, de Groot P G. Coagulation screen is more specific than the anticardiolipin antibody ELISA in defining a thrombotic $\omega$ subset of lupus patients. Ann Rheum Dis 1988; 47: 364-71. 2 Harris E N, Chan J K H, Asherson R A, Aber V R, Gharavi A E, Hughes G R V. Thrombosis, recurrent fetal loss, and thrombocytopenia: predictive value of the anticardiolipin $\mathbb{D}$ antibody test. Arch Intern Med 1986; 146: 2153-6.

3 Harris E N, Gharavi A E, Patel S P, Hughes G R V. Evaluation $T$ of the anticardiolipin antibody test: report of an international workshop held 4 April 1986. Clin Exp Immumol 1987; 68: त) 215-22.

4 Harris $E$ N, Hughes $G \quad R \quad V$. Third International Antiphospholipid Conference. Ann Rheum Dis 1988; 47: 612-4.

5 Hughes G R V. Thrombosis, abortion, cerebral disease and the lupus anticoagulant. $B r$ Med J 1983; 287: 1088-9. 
SIR, We completely agree with Dr E N Harris that carrying out clotting assays to detect lupus anticoagulant is more difficult than an enzyme linked immunosorbent assay (ELISA) for anticardiolipin antibodies. Specialised laboratories are necessary, coagulation tests are time consuming, and improper preparation and handling of plasma samples strongly influence the results of lupus anticoagulant assays.

The goal of our article was not to propose the determination of lupus anticoagulant only and to sweep aside the anticardiolipin antibody determination but to emphasise that testing anticardiolipin antibodies alone is not enough to identify patients with increased risk of thrombotic complications. From the values we found for sensitivity and specificity with lupus anticoagulant and anticardiolipin assays we conclude that in patients with systemic lupus erythematosus (SLE) the lupus anticoagulant assays correlate better with the presence of a history of thrombotic complications. We did not claim that the anticardiolipin antibody ELISA is not a useful predictor for thrombosis, fetal loss, or thrombocytopenia, but that lupus anticoagulant tests are better predictors. Confirmation of our results by studies in other laboratories is necessary, however. Therefore we endorse the last sentence of Dr Harris's letter completely that 'with the knowledge we have today both tests have to be performed'.

As mentioned by Dr Harris our studies differ with respect to the percentage of lupus patients included and the selection of patients. We evaluated 111 consecutively seen lupus patients who were unselected apart from the fact that they were seen at a university hospital. Dr Harris selected on the availability of clinical notes 121 patients (76\% with SLE) from a total of 300 patients with various autoimmune diseases (60\% with SLE) who had been screened for IgG anticardiolipin antibody levels.

In our paper we incorrectly used the term exchange of sera when we referred to the freeze dried samples which were kindly provided by Dr Harris for participation in the International Anticardiolipin Standardisation Workshop (April 1986). Although we introduced some modifications, our assay appeared to be valid. The limits we gave in units for low, medium, and high levels were obtained by transformation of the known anticardiolipin antibody concentrations (in $\mu \mathrm{g} / \mathrm{ml}$ ) in the provided samples.

We agree that apart from standardisation of the anticardiolipin antibody ELISA an international consensus with respect to the definition of the lupus anticoagulant is also urgently needed. Unless we can be sure that only results that are obtained with valid assays performed on proper samples are presented, methodological differences remain a possible cause for conflicting reports. Therefore, we welcome and strongly support the tremendous efforts made by Dr Harris and coworkers to reach international uniformity.

Departments of ${ }^{1}$ Internal Medicine (Division of Immunopathology), P HASSELAAR 13 ${ }^{2}$ Clinical Immunology, and ${ }^{3}$ Hematology, $\quad$ L BLOKZIJL $^{1} 3$ University Hospital, Utrecht, The Netherlands

\section{Cyclosporin for dermatomyositis?}

SIR, Cyclosporin was successfully used to treat adult dermatomyositis by Zabel et al. ${ }^{1}$ More recently, this drug was used to treat a patient with severe adult dermatomyositis with good effect. ${ }^{2}$ Here we present a case of severe adult dermatomyositis unresponsive to both cyclosporin and conventional treatment. In addition to redressing the balance, our case highlights other important aspects of this interesting disease.

A 67 year old woman was admitted for investigation of weakness and malaise of four months' duration. She had a heliotrope periorbital rash, marked proximal muscle weakness and wasting, Gottron's papules, and nail fold vasculitis. She was mildly anaemic, with raised inflammatory indices (Fig. 1). Electromyography indicated myositis. Left quadriceps muscle biopsy showed perifascicular fibre damage and a perivascular lymphocytic infiltrate. Serum creatine phosphokinase (CPK) was normal, as it is in $4-18 \%$ of patients with dermatomyositis at presentation, ${ }^{3}$ but lactate dehydrogenase was mildly raised, and she met the criteria for 'definite' dermatomyositis. ${ }^{4}$ Antinuclear antibody titre was weakly positive $(1 / 40)$ as was smooth muscle antibody, but rheumatoid factor and anti-Jo-1antibody were absent from her serum. Complement factors C3 and C4 were normal.

Prednisolone and azathioprine had a favourable but temporary effect after two months, when her steroid requirement fell, but she relapsed after an infection and subsequently failed to respond to increased corticosteroid

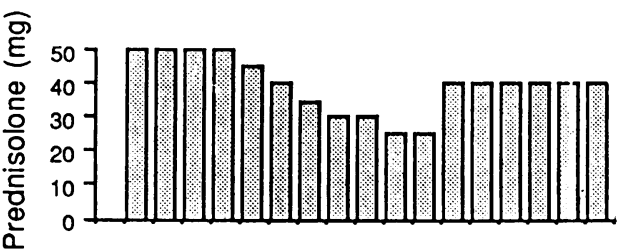

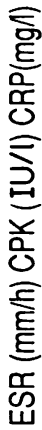

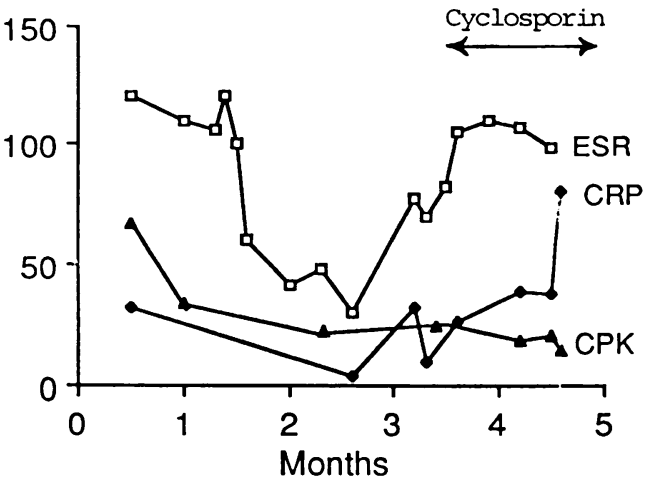

Fig. 1 Clinical course of patient with adult dermatomyositis. $E S R=$ erythrocyte sedimentation rate; $C P K=$ creatine phosphokinase; $C R P=C$ reactive protein. 